PROCEEDINGS OF THE

AMERICAN MATHEMATICAL SOCIETY

Volume 140, Number 6, June 2012, Pages 1947-1956

S 0002-9939(2011)11047-5

Article electronically published on September 23, 2011

\title{
HOMEOMORPHISMS BETWEEN LIMBS OF THE MANDELBROT SET
}

\author{
DZMITRY DUDKO AND DIERK SCHLEICHER
}

(Communicated by Bryna Kra)

\begin{abstract}
We prove that for every hyperbolic component of the Mandelbrot set, any two limbs with equal denominators are homeomorphic so that the homeomorphism preserves periods of hyperbolic components. This settles a conjecture on the Mandelbrot set that goes back to 1994 .
\end{abstract}

\section{INTRODUCTION}

The Mandelbrot set $\mathcal{M}$ is a set with a very rich combinatorial, topological, and geometric structure. It is often called "self-similar" because there are countably many dynamically defined homeomorphisms from $\mathcal{M}$ into itself, and the set of such homeomorphisms forms a semigroup. Moreover, there are many dynamically defined homeomorphisms from certain dynamically defined subsets of $\mathcal{M}$ to other subsets of $\mathcal{M}$. Perhaps the first such result was a homeomorphism from the $1 / 2$ limb of $\mathcal{M}$ to a subset of the $1 / 3$-limb of $\mathcal{M}$ constructed by Branner and Douady $\mathrm{BD}$; this class of homeomorphisms was later extended by Riedl [Ri].

In [BF1], it was shown, using homeomorphisms to parameter spaces of certain higher degree polynomials, that any two limbs $\mathcal{L}_{p / q}$ and $\mathcal{L}_{p^{\prime} / q}$ (with equal denominators) were homeomorphic. These homeomorphisms preserve the embedding into the plane so that they even extend to neighborhoods of these limbs within $\mathbb{C}$, preserving the orientation $[\mathrm{BF} 2$. All these homeomorphisms are constructed by quasiconformal surgery, and they all change the dynamics of the associated polynomials so that, in general, periods of hyperbolic components are changed.

At about the same time, it was observed [LS that there is a combinatorially defined bijection between the limbs $\mathcal{L}_{p / q}$ and $\mathcal{L}_{p^{\prime} / q}$ that preserves periods of hyperbolic components, and it was conjectured that this would yield a homeomorphism between these limbs that preserved periods of hyperbolic components. An early attempt to prove this conjecture by quasiconformal surgery resulted in another proof of the theorem from [BF1] that stayed within the quadratic family.

A proof of this conjecture is the main result of the present paper; it can be stated as follows.

Received by the editors September 7, 2010 and, in revised form, January 28, 2011.

2010 Mathematics Subject Classification. Primary 30D05, 37F10, 37F45; Secondary 37F25.

The authors gratefully acknowledge support by the Deutsche Forschungsgemeinschaft to the first author in the context of the Research Training Group 1493.

(C)2011 American Mathematical Society Reverts to public domain 28 years from publication 
Main Theorem. For any hyperbolic component of $\mathcal{M}$, let $\mathcal{L}_{p / q}$ and $\mathcal{L}_{p^{\prime} / q}$ be two limbs with equal denominators. Then there exists a homeomorphism between them that preserves periods of hyperbolic components.

Since our homeomorphism preserves periods of hyperbolic components, it can not extend to neighborhoods of the limbs.

For a fixed $n \geq 1$ consider the arrangement $\mathcal{M}_{n}$ of all hyperbolic components with periods up to $n$ (see Figure 1 for an example). There is a combinatorial model $\mathcal{M}_{\text {comb }}$ of the Mandelbrot set that can be described as a limit of $\mathcal{M}_{n}$ in a certain sense Do. Furthermore, there is a canonical continuous projection $\pi$ : $\mathcal{M} \rightarrow \mathcal{M}_{\text {comb }}$, and any fiber $\pi^{-1}(c)$ is compact, connected, and full (a bounded set $X \subset \mathbb{C}$ is called full if its complement has no bounded components). The famous "MLC conjecture" ("the Mandelbrot set is locally connected") can be stated as saying that $\pi$ is a homeomorphism.

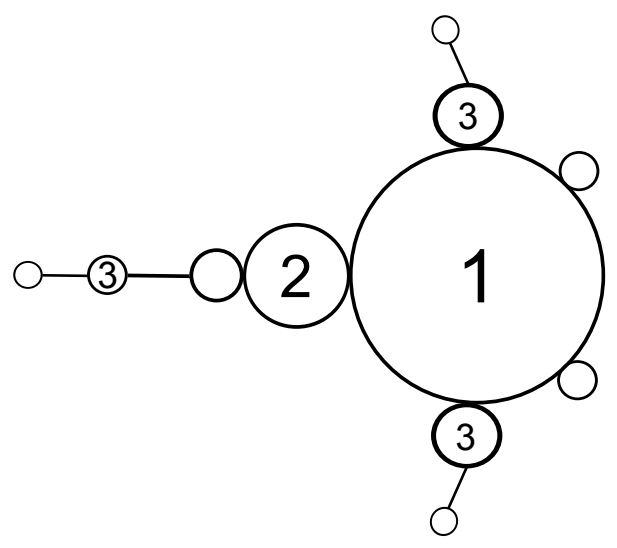

Figure 1. Combinatorics of hyperbolic components of $\mathcal{M}$ up to period 4; the hyperbolic components without numbers correspond to period 4 .

For any $p / q$ and $p^{\prime} / q$ there is a canonical homeomorphism $f^{\prime}$ between $\pi\left(\mathcal{L}_{p / q}\right)$ and $\pi\left(\mathcal{L}_{p^{\prime} / q}\right)$ preserving periods of hyperbolic components. Our strategy is to show that $f^{\prime}$ can be lifted up to the level of the Mandelbrot set; namely, we have the following commutative diagram:

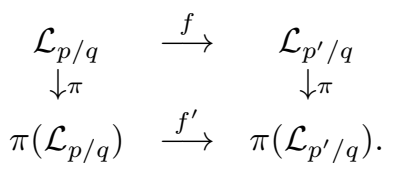

We will show that this technique can be applied to any continuous map that "respects" small copies of the Mandelbrot set.

This result fits into the vision of Douady expressed by the statement that "combinatorics implies topology": many results about the Mandelbrot sets are discovered and described in terms of combinatorics, and these combinatorial results lead the way for topological statements. In our case, the combinatorial result has remained a topological conjecture since approximately 1994. The key progress that was required was the Decoration Theorem (see below). 
Outline of the paper. In Section 2 we recall the notion of hyperbolic components, small copies of the Mandelbrot set, and combinatorial classes. The combinatorial model $\mathcal{M}_{\text {comb }}$ is defined as the quotient of $\mathcal{M}$.

Section 3 contains the definition and main properties of internal and angled internal addresses. They are coordinates for combinatorial classes.

In Section (4) we will construct diagram (1). The homeomorphism $f^{\prime}: \pi\left(\mathcal{L}_{p / q}\right) \rightarrow$ $\pi\left(\mathcal{L}_{p^{\prime} / q}\right)$ exists by fundamental properties of angled internal addresses. As $f^{\prime}$ coincides with the canonical homeomorphism on every small copy of the Mandelbrot set there exists a bijection $f$ that makes diagram (1) commute. The continuity of $f$ follows from Yoccoz's results, the existence of the canonical isomorphism of all copies of the Mandelbrot set, and the Decoration Theorem.

In Section 5 we will formulate a general statement that allows us to lift a continuous map from the level of the combinatorial model of the Mandelbrot set to the actual Mandelbrot set.

\section{The Mandelbrot Set}

The Mandelbrot set $\mathcal{M}$ is defined as the set of quadratic polynomials

$$
p_{c}: z \mapsto z^{2}+c
$$

with connected Julia sets. It is a compact, connected, and full set.

As the Mandelbrot set is the parameter space of quadratic polynomials with connected Julia sets there is an additional structure (combinatorics) of $\mathcal{M}$ on top of the topology. For instance, $\mathcal{M}$ contains hyperbolic components and small copies of the Mandelbrot set. Both types of subsets have dynamical meaning.

Hyperbolic components. A hyperbolic component of $\mathcal{M}$ is a connected component of the set of parameters $c \in \mathcal{M}$ so that $p_{c}$ has an attracting orbit.

Assume that $p_{c}(z)=z^{2}+c: \mathbb{C} \rightarrow \mathbb{C}$ has a non-repelling periodic cycle. This means there is a periodic orbit $z_{c}$ of $p_{c}$ with multiplier of absolute value at most 1. The periodic orbit $z_{c}$ is necessarily unique; let $\lambda\left(z_{c}\right)$ be its multiplier. Then it is known that:

- there is a hyperbolic component $\mathcal{H}$ such that $c \in \overline{\mathcal{H}} \subset \mathcal{M}$;

- the attracting orbit $z_{c}$ has constant period throughout $\mathcal{H}$;

- within $\mathcal{H}$ the cycle $z_{c}$ moves holomorphically;

- the multiplier map $\lambda: \mathcal{H} \rightarrow \mathbb{D}$ is a conformal isomorphism; this map extends to a homeomorphism from $\overline{\mathcal{H}}$ to $\overline{\mathbb{D}}$.

By definition, the period of $\mathcal{H}$ is the period of $z_{c}$. For every fixed $n \geq 1$ there are finitely many hyperbolic components with period $n$. The arrangement of hyperbolic components up to period $n$ gives an approximation (topological and combinatorial) to the Mandelbrot set (see Figure 1). The unique hyperbolic component of period 1 is called the main hyperbolic component of the Mandelbrot set.

Let $\mathcal{H}_{1}$ and $\mathcal{H}_{2}$ be hyperbolic components with periodic orbits $z_{c}$ and $z_{c}^{\prime}$ and periods $n_{1}$ and $n_{2}$, respectively. If the closures of $\mathcal{H}_{1}$ and $\mathcal{H}_{2}$ intersect, then the intersection is one point. Let us assume that $\mathcal{H}_{1}$ and $\mathcal{H}_{2}$ intersect, $n_{1} \leq n_{2}$, and the point $c^{\prime}$ is the intersection.

It is known that:

- $n_{2} / n_{1}=q$ is an integer greater than 1 ; 
- at $c^{\prime}$ the cycles $z_{c}$ and $z_{c}^{\prime}$ collide - every point from the cycle $z_{c}$ collides with $q$ points from the cycle $z_{c}^{\prime}$;

- the multiplier of $z_{c}$ at $c^{\prime}$ is $\exp (2 \pi i p / q)$, where $p$ is an integer coprime (and less than) to $q$; in particular, the unique non-repelling orbit of $p_{c^{\prime}}$ is parabolic.

The closure of the connected component of $\mathcal{M} \backslash \mathcal{H}_{1}$ containing $\mathcal{H}_{2}$ is called the $p / q$-limb $\mathcal{L}_{p / q}\left(\mathcal{H}_{1}\right)$ of $\mathcal{H}_{1}$. It is known that $p / q$-limbs exist for all $p / q$ in lowest terms with $q \geq 2$, and (the closure of) every component of $\mathcal{M} \backslash \mathcal{H}_{1}$ that does not contain the point $c=0$ is a limb of $\mathcal{H}_{1}$. If $\mathcal{H}_{1}$ is the main hyperbolic component, then $\mathcal{L}_{p / q}\left(\mathcal{H}_{1}\right)$ is called the (primary) $p / q$-limb $\mathcal{L}_{p / q}$.

We define the combinatorial class $\widehat{\mathcal{H}}$ of a hyperbolic component $\mathcal{H}$ as $\{c \in$ $\left.\overline{\mathcal{H}} \mid \lambda\left(z_{c}\right) \neq \exp (2 \pi i p / q), q>1\right\}$, where $\lambda\left(z_{c}\right)$ is the multiplier of the (unique) non-repelling periodic cycle $z_{c}$ of a polynomial $p_{c}$; equivalently,

$$
\widehat{\mathcal{H}}=\overline{\mathcal{H}} \backslash \bigcup_{q=2}^{\infty} \bigcup_{p} \mathcal{L}_{p / q}(\mathcal{H})
$$

Small copies of the Mandelbrot set. The Mandelbrot set is a self-similar set in the following sense: there are countably many copies of the Mandelbrot set in $\mathcal{M}$; every such copy is canonically homeomorphic to $\mathcal{M}$, where the homeomorphism is given by the straightening theorem $[\mathrm{DH}]$. In particular, small copies are compact, connected, full sets, and they preserve hyperbolic components: if a small copy intersects a hyperbolic component, then it contains that hyperbolic component. Polynomials within small copies of $\mathcal{M}$ are called renormalizable; polynomials within infinitely many nested small copies of $\mathcal{M}$ are called infinitely renormalizable.

Small copies are in one-to-one correspondence with hyperbolic components: for every hyperbolic component $\mathcal{H}$, there is a unique small copy $\mathcal{M}_{\mathcal{H}} \supset \mathcal{H}$ so that the canonical homeomorphism of $\mathcal{M}_{\mathcal{H}}$ sends $\mathcal{H}$ to the main hyperbolic component of $\mathcal{M}$ and every small copy of $\mathcal{M}$ is of this type for a unique component $\mathcal{H}$. For a small copy $\mathcal{M}_{\mathcal{H}}$, let $\operatorname{per}\left(\mathcal{M}_{\mathcal{H}}\right)$ be the period of $\mathcal{H}$. Then the canonical homeomorphism from $\mathcal{M}_{\mathcal{H}}$ to $\mathcal{M}$ divides all periods of hyperbolic components by $\operatorname{per}\left(\mathcal{M}_{\mathcal{H}}\right)$.

If $\mathcal{M}_{\mathcal{H}}$ is a small copy of $\mathcal{M}$ within $\mathcal{M}$, then $\mathcal{M} \backslash \mathcal{M}_{\mathcal{H}}$ consists of countably many connected components. The component containing the main hyperbolic component of the Mandelbrot set is the "biggest" one; all other components are called "decorations" of $\mathcal{M}_{\mathcal{H}}$. If $\mathcal{D}$ is the closure of any such decoration, then $\mathcal{D} \cap \mathcal{M}_{\mathcal{H}}$ is a single Misiurewicz point (i.e., a parameter for which the critical orbit is strictly preperiodic). The following theorem was recently proved $[\mathrm{Du}]$ (see $[\mathrm{PR}]$ for a different proof); it will be the fundamental motor for our theorem.

Decoration Theorem. For any $\varepsilon>0$, there are at most finitely many connected components of $\mathcal{M} \backslash \mathcal{M}_{\mathcal{H}}$ with diameter at least $\varepsilon$.

Yoccoz polynomials. A Yoccoz polynomial is a quadratic polynomial in $\mathcal{M}$ for which all periodic orbits are repelling, and it is not infinitely renormalizable (equivalently, it does not belong to any hyperbolic component and is not within infinitely many small copies of $\mathcal{M}$ ). It is known that $\mathcal{M}$ is locally connected at Yoccoz polynomials and, stronger yet, that the corresponding fibers of $\mathcal{M}$ are trivial. This was shown in detail in $[\mathrm{Hu}$. for non-renormalizable parameters, but results of this kind are automatically preserved by finite renormalizations [Sch2]. 
Combinatorial classes. A combinatorial class is an equivalence class of parameters with the same rational lamination. A combinatorial class is either:

- a hyperbolic combinatorial class (as defined above) or

- the intersection of an infinite nested sequence of small copies of $\mathcal{M}$ or

- a single point that does not belong to any combinatorial class of the first two types.

Any non-hyperbolic combinatorial class is always a compact, connected, and full set. A combinatorial class of the last type is exactly a Yoccoz parameter.

There are two famous conjectures: "The Mandelbrot set is locally connected" (MLC) and "hyperbolic dynamics is dense in the space of quadratic polynomials" (the Fatou conjecture for quadratic polynomials). These are equivalent to the statements "every non-hyperbolic combinatorial class is a point" and "every nonhyperbolic combinatorial class has no interior point", respectively [Do].

The combinatorial model of the Mandelbrot set. Let us say that two points $c_{1}$ and $c_{2}$ are combinatorially equivalent if $c_{1}$ and $c_{2}$ are in the same non-hyperbolic combinatorial class. The combinatorial model $\mathcal{M}_{\text {comb }}$ of the Mandelbrot set is the quotient of $\mathcal{M}$ by the above equivalence relation. The associated projection $\pi: \mathcal{M} \rightarrow \mathcal{M}_{\text {comb }}$ is called canonical.

It is known that $\mathcal{M}_{\text {comb }}$ is a connected, locally connected, compact, full set; $\pi$ is a continuous surjection; and $\pi$ is a homeomorphism (i.e., injective) if and only if MLC is valid [Do].

Hyperbolic components and small copies for $\mathcal{M}_{c o m b}$ are defined using the projection $\pi$.

Yoccoz' theorem can be expressed as follows: if $c_{n}$ is a sequence of parameters in $\mathcal{M}$ so that $\pi\left(c_{n}\right) \rightarrow \pi(c)$ for some Yoccoz parameter $c \in \mathcal{M}$, then $c_{n} \rightarrow c$, and this is the statement we need. (Note that this does not follow from the fact that $\mathcal{M}$ is locally connected at $c$. The stronger property is required that the fiber of $\mathcal{M}$ at $c$ is trivial, and Yoccoz indeed proves that; see [Sch2].)

\section{Internal ADDResses of the Mandelbrot SeT}

In this section we recall the definition and main properties of internal and angled internal addresses. The main reference is Sch1]. The motivation of an internal address is to approximate any combinatorial class by a canonical sequence of (simpler) hyperbolic classes. Internal addresses (and angled internal addresses) are defined for combinatorial classes; hence there is no difference in the definitions for $\mathcal{M}$ and $\mathcal{M}_{\text {comb }}$.

Consider a combinatorial class $C$ and a hyperbolic component $\mathcal{H}$. Assume that either $\widehat{\mathcal{H}}=C$ or $C$ is not in the connected component of $\mathcal{M} \backslash C$ containing 0 . Then we say that $\mathcal{H}$ is closer to 0 than $C$ and write $\mathcal{H} \leq C$. We also write $C<\mathcal{H}$ if $C \leq \mathcal{H}$ and $\widehat{\mathcal{H}} \neq C$.

For any $C$ inductively define the (finite or infinite) sequence

$$
\mathcal{H}_{0}<\mathcal{H}_{1}<\cdots<\mathcal{H}_{n}<\cdots
$$

such that $\mathcal{H}_{0}$ is the main hyperbolic component of $\mathcal{M}$ and $\mathcal{H}_{n}$ is of the smallest period satisfying $\mathcal{H}_{n-1}<\mathcal{H}_{n} \leq C$. For any $C$ the sequence in (4) is unique [Sch1].

Definition 3.1. Define $S_{n}$ to be the period of $\mathcal{H}_{n}$ and let $p_{n} / q_{n}$ be the fraction so that $\mathcal{H}_{n+1} \subset \mathcal{L}_{p_{n} / q_{n}}\left(\mathcal{H}_{n}\right)$. 
The sequence

$$
1=S_{0} \rightarrow S_{1} \rightarrow S_{2} \rightarrow \cdots
$$

is called the internal address of $C$.

The sequence

$$
\left(S_{0}\right)_{p_{0} / q_{0}} \rightarrow\left(S_{1}\right)_{p_{1} / q_{1}} \rightarrow\left(S_{2}\right)_{p_{2} / q_{2}} \rightarrow \cdots
$$

is called the angled internal address of $C$.

It is known [Sch1, Theorem 1.10] that an angled internal address uniquely describes a combinatorial class, where finite addresses correspond to hyperbolic classes. On the other hand the internal address describes a combinatorial class up to "symmetry". For example, hyperbolic polynomials have the same internal addresses if and only if the dynamics of the polynomials on the Julia sets are topologically conjugate. This topological conjugation extends to a neighborhood of the Julia set, preserving the orientation, if and only if the two polynomials have the same angled internal address.

Internal addresses are strictly increasing (finite or infinite) sequences of integers starting with 1 . Not every such sequence occurs for a combinatorial class of the Mandelbrot set: those that occur are called "complex admissible". An explicit characterization of the complex admissible sequences was given in [BS].

The following theorem shows the "valency of the symmetry":

Theorem 3.2 ([Sch1, Theorem 2.3]). If an angled internal address describes a combinatorial class in the Mandelbrot set, then the numerators $p_{k}$ can be changed arbitrarily (coprime to $q_{k}$ ) and the modified angled internal address still describes a combinatorial class in the Mandelbrot set.

In other words, complex admissibility is a property of internal addresses, not of angled internal addresses. In fact, any internal address uniquely determines the denominators $q_{k}$ of any associated angled internal address, while Theorem 3.2 says that the numerators $p_{k}$ are completely arbitrary (coprime to $q_{k}$ ).

Consider a small copy $\mathcal{M}_{\mathcal{H}}$ of the Mandelbrot set, where $\mathcal{H}$ is the main hyperbolic component of $\mathcal{M}_{\mathcal{H}}$. Then $\mathcal{H}$ has a finite angled internal address:

$$
\left(S_{0}^{\prime}\right)_{p_{0}^{\prime} / q_{0}^{\prime}} \rightarrow\left(S_{1}^{\prime}\right)_{p_{1}^{\prime} / q_{1}^{\prime}} \rightarrow\left(S_{2}^{\prime}\right)_{p_{2}^{\prime} / q_{2}^{\prime}} \rightarrow \cdots \rightarrow S_{n}^{\prime} .
$$

Theorem 3.3 ([Sch1, Proposition 2.7]). A combinatorial class $C$ belongs to the small copy $\mathcal{M}_{\mathcal{H}}$ with internal address as in (7) if and only if the angled internal address of $C$ is

$$
\left(S_{0}^{\prime}\right)_{p_{0}^{\prime} / q_{0}^{\prime}} \rightarrow \cdots\left(S_{n-1}^{\prime}\right)_{p_{n-1}^{\prime} / q_{n-1}^{\prime}} \rightarrow\left(S_{n}^{\prime}\right)_{p_{n} / q_{n}} \rightarrow\left(S_{n+1}\right)_{p_{n+1} / q_{n+1}} \rightarrow \cdots
$$

and $S_{n}^{\prime} \mid S_{n+k}$ for $k \geq 1$. The canonical homeomorphism between $\mathcal{M}_{\mathcal{H}}$ and $\mathcal{M}$ sends $C$ to the combinatorial class with the internal address

$$
(1)_{p_{n} / q_{n}} \rightarrow\left(S_{n+1} / S_{n}^{\prime}\right)_{p_{n+1} / q_{n+1}} \rightarrow\left(S_{n+2} / S_{n}^{\prime}\right)_{p_{n+1} / q_{n+1}} \rightarrow \cdots .
$$

We need one more result from [Sch1].

Theorem 3.4 ([Sch1, Proposition 2.6]). Consider a hyperbolic component $\mathcal{H}$ and a combinatorial class $C$ in the $p / q$-limb of $\mathcal{H}$. If $q \geq 3$, then $\mathcal{H}$ occurs in the internal address of $C$. More precisely, the internal address of $\mathcal{H}$ is a finite initial sequence of the internal address of $C$. 
This result can be expressed as follows: for a given combinatorial class $C$, there are usually many hyperbolic components $\mathcal{H}<C$, and most of them are not associated to the internal address of $C$. For those that are not, $C$ is in the $1 / 2$-limb of $\mathcal{H}$.

\section{Proof of The HomeOMORPhism theOREM}

In this section, we prove the main theorem in an apparently stronger form: consider hyperbolic components $\mathcal{H}_{1}$ and $\mathcal{H}_{2}$ with identical internal addresses. Then we will construct a homeomorphism between the $\operatorname{limbs} \mathcal{L}_{p / q}\left(\mathcal{H}_{1}\right)$ and $\mathcal{L}_{p^{\prime} / q}\left(\mathcal{H}_{2}\right)$, where $q \geq 3$. The original statement of the Main Theorem describes the case $\mathcal{H}_{1}=\mathcal{H}_{2}$.

Remark. This more general version can easily be deduced from the statement of the Main Theorem, because there is a unique hyperbolic $\mathcal{H}^{\prime}$ at which the angled internal addresses of $\mathcal{H}_{1}$ and $\mathcal{H}_{2}$ branch off, in the sense that $\mathcal{H}_{1}$ and $\mathcal{H}_{2}$ are contained in two different limbs at angles $p / q$ and $p^{\prime} / q$ of $\mathcal{H}^{\prime}$, with $q \geq 3$. A possibly repeated application of the Main Theorem will then yield the statement we are proving here, and it shows that the statement remains true for $q=2$; i.e., for the $\operatorname{limbs} \mathcal{L}_{1 / 2}\left(\mathcal{H}_{1}\right)$ and $\mathcal{L}_{1 / 2}\left(\mathcal{H}_{2}\right)$. The reason why we are giving an apparently more general proof is because the proof really is the same, and this illustrates the general nature of the argument.

Let the angled internal addresses of $\mathcal{H}_{1}$ and $\mathcal{H}_{2}$ be

$$
\begin{gathered}
\left(S_{0}\right)_{p_{0} / q_{0}} \rightarrow \cdots \rightarrow\left(S_{n-1}\right)_{p_{n-1} / q_{n-1}} \rightarrow\left(S_{n}\right), \\
\left(S_{0}\right)_{p_{0}^{\prime} / q_{0}} \rightarrow \cdots \rightarrow\left(S_{n-1}\right)_{p_{n-1}^{\prime} / q_{n-1}} \rightarrow\left(S_{n}\right)
\end{gathered}
$$

respectively. By Theorem 3.4 the limbs $\mathcal{L}_{p / q}\left(\mathcal{H}_{1}\right)$ and $\mathcal{L}_{p^{\prime} / q}\left(\mathcal{H}_{2}\right)$ consist exactly of all combinatorial classes that have internal addresses starting with

$$
\begin{aligned}
& \left(S_{0}\right)_{p_{0} / q_{0}} \rightarrow \cdots \rightarrow\left(S_{n-1}\right)_{p_{n-1} / q_{n-1}} \rightarrow\left(S_{n}\right)_{p / q} \rightarrow \\
& \left(S_{0}\right)_{p_{0}^{\prime} / q_{0}} \rightarrow \cdots \rightarrow\left(S_{n-1}\right)_{p_{n-1}^{\prime} / q_{n-1}} \rightarrow\left(S_{n}\right)_{p^{\prime} / q} \rightarrow
\end{aligned}
$$

respectively.

Define a map $f^{\prime}: \pi\left(\mathcal{L}_{p / q}\left(\mathcal{H}_{1}\right)\right) \rightarrow \pi\left(\mathcal{L}_{p^{\prime} / q}\left(\mathcal{H}_{2}\right)\right)$ so that it changes the initial segment (12) of the angled internal address into the segment (13); i.e., it changes the angles in the angled internal address from the limb $\mathcal{L}_{p / q}\left(\mathcal{H}_{1}\right)$ into the limb $\mathcal{L}_{p^{\prime} / q}\left(\mathcal{H}_{2}\right)$. Within hyperbolic components, this map should fix multipliers. It follows from the construction and Theorem 3.2 that the map $f^{\prime}$ is a well-defined homeomorphism and preserves internal addresses. We will show that there exists a canonical homeomorphism $f$ such that

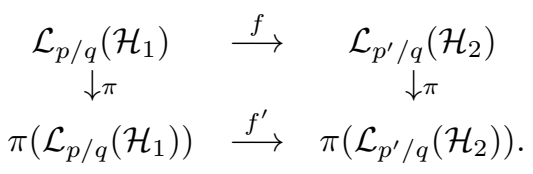

By canonical we mean that $f$ coincides with the natural homeomorphism between small copies of the Mandelbrot set. With this requirement there is a unique bijection $f$ that makes the above diagram commute. Indeed, if a non-hyperbolic 
combinatorial class $C$ does not belong to any small copy of $\mathcal{M}$, then $C$ is a point and $f(C)$ is uniquely defined. If $C$ belongs to a small copy $\mathcal{M}_{\mathcal{H}} \subset \mathcal{L}_{p / q}\left(\mathcal{H}_{1}\right)$ of $\mathcal{M}$, then $f$ on $\mathcal{M}_{\mathcal{H}}$ is uniquely defined by the requirement that $f$ be canonical (note that $f^{\prime}$ is canonical by Theorem 3.3.

The main issue is to prove that $f$ is continuous. Let us prove that if $c_{n}$ tends to $c_{\infty}$, then $f\left(c_{n}\right)$ tends to $f\left(c_{\infty}\right)$. This will imply that $f$ is a homeomorphism (as $f$ is a continuous bijection between compact Hausdorff spaces). It suffices to consider the following three cases.

Case 1 . Assume $c_{\infty}$ belongs to at most finitely many small copies of the Mandelbrot set; then the same is true for $f\left(c_{\infty}\right)$. By construction, $f^{\prime}\left(\pi\left(c_{n}\right)\right)=\pi\left(f\left(c_{n}\right)\right)$ tends to $f^{\prime}\left(\pi\left(c_{\infty}\right)\right)=\pi\left(f\left(c_{\infty}\right)\right)$ (using commutativity of diagram (14) $)$. By Yoccoz' theorem, it follows that $f\left(c_{n}\right)$ tends to $f\left(c_{\infty}\right)$.

Case 2. Assume $c_{\infty}$ and all $c_{n}$ belong to a single small copy $\mathcal{M}_{\mathcal{H}}$, where $\mathcal{M}_{\mathcal{H}} \subset$ $\mathcal{L}_{p / q}\left(\mathcal{H}_{1}\right)$. Then the statement follows from the definition of $f$ because $f$ coincides with the canonical homeomorphism from $\mathcal{M}_{\mathcal{H}}$ to $f\left(\mathcal{M}_{\mathcal{H}}\right)$.

Case 3. Assume $c_{\infty}$ belongs to infinitely many copies of the Mandelbrot set (i.e., $c_{\infty}$ is infinitely renormalizable), $\mathcal{M}_{\mathcal{H}} \subset \mathcal{L}_{p / q}\left(\mathcal{H}_{1}\right)$ is a small copy containing $c_{\infty}$, and $c_{n}$ does not belong to $\mathcal{M}_{\mathcal{H}}$ for any $n$.

Let $\mathcal{D}_{n}$ be the closure of the connected component of $\mathcal{M} \backslash \mathcal{M}_{\mathcal{H}}$ containing $c_{n}$, and let $a_{n}$ be the intersection of $\mathcal{M}_{\mathcal{H}}$ and $\mathcal{D}_{n}$. Then $a_{n}$ is a single Misiurewicz point and hence belongs to at most finitely many copies of $\mathcal{M}$.

As $c_{\infty}$ belongs to infinitely many copies of $\mathcal{M}$, it follows that $c_{\infty} \neq a_{k}$ for all $k$. Therefore only finitely many $c_{n}$ are in $\mathcal{D}_{k}$ for each fixed $k$. Hence by the Decoration Theorem the distance between $c_{k}$ and $a_{k}$ tends to 0 , and so the sequence $a_{k}$ tends to $c_{\infty}$. By Case 2 we obtain that $f\left(a_{k}\right)$ tends to $f\left(c_{\infty}\right)$.

By a similar reason the distance between $f\left(a_{k}\right)$ and $f\left(c_{k}\right)$ tends to $0\left(f\left(a_{k}\right)\right.$ is the intersection of $f\left(\mathcal{M}_{\mathcal{H}}\right)$ with the closure of the connected component of $\mathcal{M} \backslash \mathcal{M}_{\mathcal{H}}$ containing $f\left(c_{k}\right)$ ). We conclude that $f\left(c_{k}\right)$ tends to $f\left(c_{\infty}\right)$. This concludes the proof of the Main Theorem.

\section{Generalization}

We say that a set $\mathcal{L} \subset \mathcal{M}$ is combinatorially saturated if $\pi^{-1}(\pi(\mathcal{L}))=\mathcal{L}$. Consider two closed combinatorially saturated sets $\mathcal{L}_{1}$ and $\mathcal{L}_{2}$ and assume that there exists a continuous map $f^{\prime}: \pi\left(\mathcal{L}_{1}\right) \rightarrow \pi\left(\mathcal{L}_{2}\right)$.

We say that $f^{\prime}$ is canonical (with respect to small copies of $\mathcal{M}$ ) if:

- for every infinitely renormalizable $c \in \pi\left(\mathcal{L}_{1}\right)$ there exists a copy $\pi\left(\mathcal{M}_{\mathcal{H}}\right) \subset$ $\pi\left(\mathcal{L}_{1}\right)$ containing $c$ such that $f^{\prime}$ restricted to $\pi\left(\mathcal{M}_{\mathcal{H}}\right)$ is the canonical homeomorphism on $\pi\left(\mathcal{M}_{\mathcal{H}}\right)$;

- for every infinitely renormalizable $c \in \pi\left(\mathcal{L}_{2}\right)$ there exists a copy $\pi\left(\mathcal{M}_{\mathcal{H}}\right) \subset$ $\pi\left(\mathcal{L}_{2}\right)$ containing $c$ such that $f^{\prime}$ restricted to any connected component of $f^{\prime-1}\left(\pi\left(\mathcal{M}_{\mathcal{H}}\right)\right)$ is the canonical homeomorphism of a small copy of the Mandelbrot set.

In particular, by a standard compactness argument $f^{\prime-1}\left(\pi\left(\mathcal{M}_{\mathcal{H}}\right)\right.$ ) (in the second condition) consists of finitely many small copies. 
Theorem 5.1. Let $\mathcal{L}_{1}, \mathcal{L}_{2} \subset \mathcal{M}$ be two closed connected combinatorial sets. For every continuous map $f^{\prime}: \pi\left(\mathcal{L}_{1}\right) \rightarrow \pi\left(\mathcal{L}_{2}\right)$ that is canonical with respect to small copies of the Mandelbrot set, there exists a continuous map $f: \mathcal{L}_{1} \rightarrow \mathcal{L}_{2}$ such that the following diagram commutes:

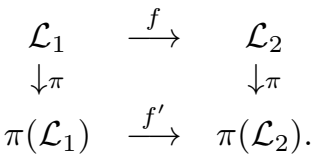

The proof is quite similar to the previous one and is left to the reader.

\section{ACKNOWLEDGEMENT}

The authors would like to thank Misha Lyubich for helpful and pleasant discussions over the years.

\section{REFERENCES}

[ALS] A. Avila, M. Lyubich and W. Shein, Parapuzzle of the Multibrot set and typical dynamics of unimodal maps. Journal of the European Mathematical Society, 13 (2011), no. 1, 27-56. MR2735075

[BD] B. Branner, A. Douady, Surgery on complex polynomials. Holomorphic Dynamics (Mexico, 1986), 11-72, Lecture Notes in Math., 1345, Springer, Berlin, 1988. MR980952 (90e:58114)

[BF1] B. Branner, N. Fagella, Homeomorphisms between limbs of the Mandelbrot set. Journal of Geom. Anal. 9 (1999), 327-390. MR1757453 (2001j:37082)

[BF2] B. Branner, N. Fagella, Extension of homeomorphisms between limbs of the Mandelbrot set. Conform. Geom. and Dynam. 5 (2001), 100-139. MR1872159 (2002h:37076)

[BS] H. Bruin, D. Schleicher, Admissibility of kneading sequences and structure of Hubbard trees for quadratic polynomials. J. Lond. Math. Soc. 2 (2008), 502-522. MR2439637 (2009j:37063)

[Do] A. Douady, Descriptions of compact sets in $\mathcal{C}$. In: Topological Methods in Modern Mathematics (Stony Brook, NY, 1991), Publish or Perish, Houston, TX (1993), 429-465. MR.1215973 (94g:58185)

[DH] A. Douady, J.H. Hubbard, On the dynamics of polynomial-like maps. Ann. Sci. Ècole Norm. Sup. 18 (1985), 287-343. MR816367 (87f:58083)

[Du] D. Dudko, The decoration theorem for Mandelbrot and Multibrot sets. Preprint, arXiv:1004.0633v1 [math.DS], 2010. Submitted.

[Hu] J.H. Hubbard, Local connectivity of Julia sets and bifurcation loci: Three theorems of J.-C. Yoccoz. In: Topological Methods in Modern Mathematics, 467-511 and 375-378 (figures), Publish or Perish (1993). MR.1215974 (94c:58172)

[KL1] J. Kahn, M. Lyubich, A priori bounds for some infinitely renormalizable quadratics: II. Decorations. Ann. Sci. Ec. Norm. Sup. (4) 41 (2008), no. 1, 57-84. MR2423310 (2009k:37106)

[KL2] J. Kahn, M. Lyubich, A priori bounds for some infinitely renormalizable quadratics: III. Molecules. In: Complex Dynamics: Families and Friends (ed. D. Schleicher), 229-254, A K Peters, Wellesley, MA (2009). MR2508259 (2010f:37078)

[LS] E. Lau, D. Schleicher, Internal addresses in the Mandelbrot set and irreducibility of polynomials. Preprint 19, Institute of Mathematical Sciences, Stony Brook (1994).

[L1] M. Lyubich, Dynamics of quadratic polynomials, I-II. Acta Math. 178 (1997), 185-297. MR.1459261 (98e:58145)

[L2] M. Lyubich, Dynamics of quadratic polynomials, III. Parapuzzle and SBR measure. In: Géométrie complexe et systèmes dynamiques, Astérisque 261 (2000), 173-200. MR1755441 (2002e:37060)

[Mc] C. McMullen, Complex Dynamics and Renormalization. Ann. of Math. Stud., Princeton Univ. (1994). MR:1312365 (96b:58097) 
[Mi] J. Milnor, Periodic orbits, externals rays and the Mandelbrot set: an expository account. In: Géométrie complexe et systèmes dynamiques, Astérisque 261 (2000), 277-333. MR.1755445 (2002e:37067)

[PR] C. L. Petersen, P. Roesch, Carrots for dessert. Preprint, arXiv:1003.3947v1 [math.DS], 2010. Submitted.

[Ri] J. Riedl, Arcs in Multibrot Sets, Locally Connected Julia Sets and Their Construction by Quasiconforman Surgery. Thesis, Technische Universität München, 2001, available at the Stony Brook IMS thesis server

[Sch1] D. Schleicher, Internal addresses of the Mandelbrot set and Galois groups of polynomials. Preprint, arXiv:math/9411238v2 [math.DS], 1994.

[Sch2] D. Schleicher, On fibers and local connectivity of Mandelbrot and Multibrot sets. In: Fractal Geometry and Applications: A Jubilee of Benoit Mandelbrot. M. Lapidus, M. van Frankenhuysen (eds), Proc. Sympos. Pure Math. 72, Amer. Math. Soc. (2004), 477-517. MR2112117 (2006b:37088)

Research I, Jacobs University, Postfach 750 561, D-28725 Bremen, Germany - and G.-A.-Universität zu Göttingen, Bunsenstrasse 3-5, D-37073 Göttingen, Germany

E-mail address: d.dudko@jacobs-university.de

Research I, Jacobs University, Postfach 750 561, D-28725 Bremen, Germany

E-mail address: dierk@jacobs-university.de 